\title{
Finite element modelling of crack propagation in metal powder compaction using Mohr-Coulomb and Elliptical Cap yield criteria
}

\begin{abstract}
In the modelling of the compaction process of particulate materials into a coherent green body, an appropriate yield criterion for the deformation process has to be selected. In this paper, two commonly used yield criteria for powder compaction, namely Mohr-Coulomb and Elliptical Cap are utilized in the finite element modelling of iron powder compaction process, which incorporates a fracture criterion of granular materials in compression. The simulated crack growth patterns obtained by using these two yield criteria were compared in terms of the influence of shear stress and relative density distributions. This work has shown that the application of the Elliptical Cap yield model gives a more realistic simulated Mode II crack propagation during the densification stage of the metal powder compaction process in comparison to the Mohr-Coulomb yield model.
\end{abstract}

Keyword: Compaction, Crack propagation, Finite element, Mohr-Coulomb, Elliptical Cap, Shear crack 